\title{
Prevalence of Mycobacterium avium ssp. paratuberculosis infections in Canadian dairy herds
}

\author{
Caroline S. Corbett, ${ }^{*}$ S. Ali Naqvi, ${ }^{*}$ Cathy A. Bauman, $\nmid$ Jeroen De Buck, ${ }^{*}$ Karin Orsel, ${ }^{*}$ Fabienne Uehlinger,ł \\ David F. Kelton, $†$ and Herman W. Barkema*1 \\ *Department of Production Animal Health, Faculty of Veterinary Medicine, University of Calgary, Calgary, AB, Canada, T2N 4N1 \\ †Department of Population Medicine, Ontario Veterinary College, University of Guelph, Guelph, ON, Canada, N1G 2W1 \\ łDepartment of Large Animal Clinical Sciences, Western College of Veterinary Medicine, University of Saskatchewan, Saskatoon, SK, Canada, \\ S7N 5B4
}

\section{ABSTRACT}

Johne's disease is a progressive, chronic disease with inflammation of the small intestine of ruminants caused by Mycobacterium avium ssp. paratuberculosis (MAP). Accurately estimating prevalence of MAP infections is important when controlling spread of infection or monitoring effectiveness of control programs. In the absence of a consistent test method used in prevalence studies across Canada, prevalence estimates among regions and programs cannot be compared. The aim of the current study was to estimate and compare prevalence of MAP infection in Western Canada, Ontario, Québec, and the Atlantic provinces, as well as among varying herd sizes and housing types. On 362 dairy farms located in all 10 provinces of Canada, environmental samples were collected and cultured for detection of MAP. For each herd, 1 sample was collected from the lactating cow area and manure storage. An additional environmental sample was collected from the area where breeding-age heifers were housed. Using prior distributions from previous research, diagnostic sensitivity and specificity were calculated to assess the ability of only 2 environmental samples (manure storage and lactating cow area) to identify MAP-positive farms, resulting in a sensitivity and specificity of 38 and 100\%, respectively. We found no difference in sensitivity and specificity when including breeding-age heifers environmental samples. Test characteristics were applied to environmental culture results from the 362 participating farms in all 4 regions, resulting in true prevalence estimates of $66 \%$ for farms in Western Canada, 54\% in Ontario, 24\% in Québec, and $47 \%$ in Atlantic Canada. Herds housed in tiestalls had lower prevalence than freestall-housed herds, and

Received March 31, 2018.

Accepted August 23, 2018.

${ }^{1}$ Corresponding author: barkema@ucalgary.ca herds with 101-150 and $>151$ cows had higher prevalence than herds with $\leq 100$ cows. This was the first time MAP prevalence was determined using 1 detection method, performed in 1 laboratory, and within a single year across Canada, enabling direct comparisons of prevalence among regions, housing types, and herd sizes.

Key words: Johne's disease, prevalence, Canada, environmental samples, herd characteristics

\section{INTRODUCTION}

Johne's disease (JD) is caused by Mycobacterium avium ssp. paratuberculosis (MAP) and results in progressive chronic enteritis in most ruminant species (Cocito et al., 1994). Infection is widespread worldwide among dairy cattle, leading to economic losses due to reduced productivity and increased risk of culling (McKenna et al., 2006). In the absence of a vaccine to prevent MAP infection, or an effective treatment for infected cattle, control is primarily based on preventing new infections within and between herds. Control programs for JD in cattle have been implemented around the world and vary based on their aims, typically eradication, surveillance, certification of JD-negative herds, or decreasing transmission (Kalis et al., 2004; Bakker, 2010; Collins et al., 2010; Barkema et al., 2018).

Accurate and reliable prevalence estimates can be used to monitor success of implemented control programs or to estimate economic impacts of the disease (Barkema et al., 2010). Prevalence estimates in Canada, at animal or herd levels, are calculated based on results of screening tests for detection of MAP infection and include environmental samples, bulk tank ELISA, tissues cultured from slaughtered cattle, and serum or milk ELISA (McKenna et al., 2004; Wolf et al., 2014; Pieper et al., 2015). These tests rely on detection of the bacteria or the immune response produced by an infected animal. However, accurate diagnosis can be difficult, due to variable disease progression resulting 
in delayed immune responses, intermittent shedding of bacteria in feces and milk, and varying sensitivities (Se) and specificities (Sp) of diagnostic tests (Weber, 2006; Lombard, 2011; Barkema et al., 2018). Although culture of MAP, either in feces or tissue samples, has historically been considered the gold standard of infection detection due to high SP, limitations of poor Se and intermittent shedding have challenged this designation. Little consensus exists regarding appropriate adjustments for Se and Sp for MAP infection, as there is no gold standard or reference for detection, making comparisons of true estimates difficult (Tiwari et al., 2006).

Prevalence of MAP infection has been estimated among regions in Canada using various diagnostic tests (McKenna et al., 2004; Wolf et al., 2014; Pieper et al., 2015). Because choice of test used for detection of MAP infection affects the ability to identify infection status at both animal and herd levels and estimates of test characteristics are not always accurate, comparing prevalence across regions or time frames is often difficult and unreliable (Lombard, 2011; Barkema et al., 2018). Regardless of method used, apparent prevalence will depend on test characteristics and must be adjusted for test Se and Sp to estimate true prevalence (Whitlock et al., 2000; Garcia and Shalloo, 2015).

Herd-level prevalence within a region has been estimated using environmental sampling of herds without individual cow sampling (Wolf et al., 2014). When comparing prevalence of MAP infection across regions, it is important to not only use the same screening test method (Garcia and Shalloo, 2015), but to also account for differences in herd size and housing type, as these affect the probability of detecting MAP-infected herds (Berghaus et al., 2006; Wolf et al., 2015b). As herd size increases, the probability of a positive test increases, as does the ability to detect MAP in the environment, because more cattle are likely to be fecal shedders (van Schaik et al., 2003; Wolf et al., 2015b). Likewise, odds of herds testing MAP-positive is lower in tiestall compared with freestall herds, likely due to differences in contact structure of animals, less mixing of feces, and management practices (Wolf et al., 2014). Additionally, increasing evidence has shown that young stock shed MAP into their environment (Bolton et al., 2011; Wolf et al., 2015c). It is not known, however, whether including environmental samples from young stock housing affects identification of MAP-positive farms or resulting herd prevalence estimates. Typically, 6 environmental samples are collected from areas of manure accumulation, with no samples collected from the environment of young stock (Berghaus et al., 2006; Wolf et al., 2014). Sensitivities and Sp for detection of herds when collecting 6 environmental samples have been estimated and used for estimation of true herdlevel prevalence (Wolf et al., 2014); however, Se and Sp have not been estimated when collecting fewer samples for detecting MAP-infection status at the herd level or when including environmental samples from young stock. Decreasing the number of environmental samples used for herd detection would decrease cost and potentially enable broader surveillance across large regions.

Objectives of our study were to (1) determine Se and Sp for detecting MAP-positive farms based on 2 environmental samples, and 3 environmental samples when including an additional sample from breeding-age heifers (BAH); (2) estimate true herd-level prevalence of MAP infection across 4 regions of Canada; and (3) compare estimated apparent and true MAP prevalence across herd size categories and housing types.

\section{MATERIALS AND METHODS}

\section{Herd Selection and Surveys}

Farms included in the study were sampled at convenience as part of the Canadian National Dairy Study (NDS; Bauman et al., 2018). Briefly, producers were recruited to participate in the NDS based on information provided by the 10 provincial milk marketing boards across Canada. All licensed Canadian dairy producers were invited to participate in a national survey. From the 1,062 respondents that completed the national survey, a subset of herds was randomly selected within each province to participate in an onfarm visit for an additional survey and collection of biological samples. The number of herds selected for a farm visit was based on a sample size calculation with the following parameters: allowable error of 5\%, 95\% confidence level, and expected herd-level prevalence of $\sim 40 \%$ for common endemic diseases, including Staphylococcus aureus mastitis; collectively, this resulted in the need to include 368 farms (Bauman et al., 2018). Farms were selected to represent each region in Canada as well as to be proportional to the number of producers located in each province: Western Canada [60; British Columbia (20), Alberta (20), Saskatchewan (10), and Manitoba (10)], Ontario (120), Québec (120), and Atlantic Canada [65; New Brunswick (20), Nova Scotia (20), Prince Edward Island (20), and Newfoundland (5)] (Bauman et al., 2018). These numbers were slightly modified due to limited numbers of sampling staff in some regions and costs of travel to remote locations, resulting in 362 farms being sampled across Canada. Herd size and cow housing type were recorded at each farm visit. 


\section{Environmental Sample Collection and MAP Culture}

In total, 14 university summer students (3 sampled Western Canada, 5 sampled Ontario, 4 sampled Québec, and 2 sampled Atlantic Canada) were recruited from the 5 respective Canadian veterinary schools and underwent standardized training at the University of Guelph over 3 d (Bauman et al., 2018). At each farm visit, 2 environmental samples were collected from areas with the highest probability of finding MAP, based previous studies (Wolf et al., 2014); 1 environmental sample was collected from the area of the farm where lactating cows were housed (LAC) and 1 environmental sample from the manure storage area (MSA) or area of manure accumulation. An additional environmental sample was collected from the area where $\mathrm{BAH}$ were housed. Each environmental sample consisted of 4 subsamples collected by a technician wearing a latex glove who placed each of these $\sim 150$-g samples into a single resealable plastic bag, thoroughly mixed them, and then filled a 50-mL screw-top plastic container, as described (Wolf et al., 2014). In tiestall barns, these 4 subsamples were collected from the lactating herd in the manure gutter corners and high-traffic areas, whereas freestall samples were collected from crossover alleys and near waterers. Manure storage subsamples were collected from a manure pile using a gloved hand that reached 10 to $15 \mathrm{~cm}$ below the external surface or from a manure pit using a 1.2-m long golf ball retriever (extendable to $4.5 \mathrm{~m}$ ) with a $50-\mathrm{mL}$ plastic container taped to the end. Subsamples from the BAH pens consisted of a handful from the manure pack of every pen of heifers, or 4 handfuls from well-traveled areas if heifers were all kept in the same area, and mixed as per the lactating cow herd. All samples were stored on ice packs and sent by express mail (guaranteed 48-h delivery) to the University of Calgary (Calgary, $\mathrm{AB}$, Canada) for processing. All samples were stored at $4^{\circ} \mathrm{C}$ and processed within $7 \mathrm{~d}$ after collection using the Trek ESP II (Trek para-JEM; Trek Diagnostic Systems, Cleveland, $\mathrm{OH}$ ) liquid culture protocol and media. After 7 wk of incubation at $37^{\circ} \mathrm{C}$, DNA was extracted and detection of MAP was based on subsequent F57-specific quantitative PCR (qPCR), as described (Corbett et al., 2017). Samples with cycle threshold value $<40$ were considered positive.

\section{Statistical Analyses}

As the present method of environmental sampling had not been implemented in previous studies, first test characteristics (Se and $\mathrm{Sp}$ ) for this type of environmental sampling (1 LAC and $1 \mathrm{MSA}$ on the same day) needed to be estimated using Bayesian models, as described below. Prior Se and Sp estimate inputs were calculated based on a complete data set available from a previous study (Wolf et al., 2014) to more accurately determine true prevalence across Canada. Sensitivity, Sp, and true prevalence were estimated using $\mathrm{R}$ v3.4.2 ( $\mathrm{R}$ Core Team, 2017), the 'BetaBuster' function in the 'epiR' package (Stevenson et al., 2017), the 'rjags' package (Plummer, 2016), the 'runjags' package (Denwood, 2016), and utility programs included with Doing Bayesian Data Analysis: A Tutorial with R, JAGS and Stan (Kruschke, 2015).

Prior Test Characteristics. Test characteristics (Se and $\mathrm{Sp}$ ) were estimated based on MAP environmental sample results collected by the Alberta Johne's Disease Initiative between November 2010 and May 2014 (Wolf et al., 2014), during which time 6 environmental samples were collected from the same farms for 3 consecutive years (6S3Y). Briefly, samples were collected in duplicate from 3 areas on 430 Alberta dairy farms from (1) MSA, (2) LAC, and (3) dry, sick, or calving pens; this resulted in 6 samples per farm, processed as described above. As not all sampling records specified the location at which each environmental sample was collected, a subset of farms $(\mathrm{n}=62)$ was identified that were sampled in 3 consecutive years, and each year had at least 1 MSA and 1 LAC area sample identified. These 62 farms encompassed all herd size categories, ranging from 43 to 405 lactating cows with a mean of $74(\mathrm{SD}=74$, median $=116)$.

The Se and Sp estimates for 6S3Y were used to further determine the Se and Sp for these 62 farms when only 2 samples (MSA + LAC) were collected once $(\mathbf{2 S 1 Y})$ to simulate the NDS sampling scheme. On these 62 farms, 2 samples were randomly selected from $1 \mathrm{MSA}$ and 1 LAC area sample from a randomly selected year within the $3 \mathrm{yr}$ available for each herd. Herd-level MAP infection status was based on environmental samples from the same areas within the same herds; therefore, Se and Sp were estimated using a Bayesian model that accounted for conditional dependence between diagnostic tests (Dendukuri and Joseph, 2001). Prior distributions for Se and Sp of 6S3Y, as well as MAP prevalence, were defined as following a $\beta$ distribution whose parameters were determined by estimates from Wolf et al. (2014) using an implementation of the BetaBuster program (https://www2.vetmed.ucdavis.edu/cadms/ local_resources/docs/betabuster012006.zip) in R. Beta parameters were determined by specifying the range where the true parameter lies with $95 \%$ confidence. The $\beta$ parameters, $\alpha$ and $\beta$, can also be interpreted as scaled versions of number of true positives and false-negatives, respectively. Prior distributions for Se and Sp of 2S1Y were chosen to represent minimal prior knowledge. Specifications for prior distribution are shown in Table 
Table 1. Parameters used for prior distributions to estimate test accuracy of the environmental sampling scheme used and herd-level prevalence stratified by region, herd size, or housing type of Mycobacterium avium ssp. paratuberculosis (MAP) infection on Canadian dairy farms

\begin{tabular}{|c|c|c|c|}
\hline Parameter & Distribution type & Distribution parameter & Value \\
\hline 6S3Y specificity & & $\beta$ & 1.34 \\
\hline 2S1Y sensitivity & Uniform & $\begin{array}{l}a^{3} \\
b^{3}\end{array}$ & $\begin{array}{l}0.00 \\
1.00\end{array}$ \\
\hline \multirow[t]{2}{*}{ Alberta MAP prevalence } & Beta & $\alpha$ & 13.18 \\
\hline \multirow{2}{*}{\multicolumn{4}{|c|}{ Estimating MAP prevalence $^{4}$}} \\
\hline & & & \\
\hline \multirow[t]{2}{*}{ 2S1Y sensitivity } & Beta & $\alpha$ & 9.50 \\
\hline & & $\beta$ & 13.65 \\
\hline \multirow[t]{2}{*}{ 2S1Y specificity } & Beta & $\alpha$ & 14.40 \\
\hline & & $\beta$ & 1.12 \\
\hline Canada MAP prevalence & & $\mathrm{b}$ & 1.00 \\
\hline \multicolumn{4}{|l|}{ Region, housing type, and herd size ${ }^{5}$} \\
\hline \multirow[t]{2}{*}{ Region/housing type/herd size specific prevalence } & Gaussian & $\mu($ mean $)$ & 0.00 \\
\hline & & $\tau$ (precision) & 1.00 \\
\hline \multirow{2}{*}{ 2S1Y sensitivity } & Beta & $\alpha$ & 9.50 \\
\hline & & $\beta$ & 13.65 \\
\hline \multirow[t]{2}{*}{ 2S1Y specificity } & Beta & $\alpha$ & 14.40 \\
\hline & & $\beta$ & 1.12 \\
\hline
\end{tabular}

${ }^{1}$ Model comparing herd-level MAP-infection status based on 1 positive in 6 samples collected for 3 yr (6S3Y) to estimate based on 1 MAPpositive sample out of 2 collected in $1 \mathrm{yr}(2 \mathrm{~S} 1 \mathrm{Y})$, consisting of a manure storage (MSA) and a lactating cow area sample (LAC). Data were collected in Alberta between 2010 and 2014 (Wolf et al., 2014), so a prior distribution for prevalence also needed to be specified.

${ }^{2}$ Shape parameters for a $\beta$ distribution. In the context of sensitivity/specificity estimation $\alpha$ is proportional to the number of true positives in the sample, and $\beta$ is proportional to the number of falsenegatives.

${ }^{3}$ Lower (a) and upper (b) bounds for uniform distribution. All values in that range have equal probability.

${ }^{4}$ Model estimating prevalence based on 2 samples (MSA and LAC) collected in a single year (2S1Y), compared with sensitivity and specificity of collecting 1 additional sample from breeding-age heifers area (3S1Y).

${ }^{5}$ Model estimating regional, housing type-specifics and herd size-specific herd-level MAP-infection prevalence in Canada in 2015 based on 2 samples taken from each herd $(2 \mathrm{~S} 1 \mathrm{Y})$.

1. Sensitivity and Sp of both $6 \mathrm{~S} 3 \mathrm{Y}$ and $2 \mathrm{~S} 1 \mathrm{Y}$ were simultaneously estimated using a Gibbs sampler, and modes of the resulting posterior distributions were presented with their respective probability intervals. Gibbs samplers were run using 6 chains in parallel for a combined total of 25,000 iterations after a burn-in of 5,000 iterations. Convergence was verified by ensuring that posterior distributions were unimodal and that the Gelman and Rubin R statistic (Gelman and Rubin, 1992) reached 1 by the end of the chains.

Estimating MAP Prevalence. True herd-level MAP prevalence in dairy herds across Canada was estimated using a Bayesian model, using prior distributions of the Se and Sp as described above and based on the 362 herds sampled in 2015 (NDS; Bauman et al., 2018). The model also compared MAP status based on 2 samples (LAC and MSA samples), with MAP-status based on 3 samples (LAC, MSA, and BAH) collected in $1 \mathrm{yr}(\mathbf{3 S 1 Y})$ with estimated Se and Sp (3S1Y). The model was run for the same number of iterations as the model determining test characteristics and convergence was also monitored using the Gelman and Rubin $\mathrm{R}$ statistic (Gelman and Rubin, 1992).

Region, Housing Type, and Herd Size. The 362 NDS herds were used to estimate region, housing type, and herd-size-specific prevalence in Canada. Associations between herd size and housing type were assessed with ANOVA, followed by least squares means comparisons, with herd size being the outcome and housing type being the predictor. Stratified prevalence estimates were based on 2 samples, LAC and MSA (2S1Y). Prevalences of MAP infection specific to region, herd size, and housing type were estimated using logistic regression models applied in a Bayesian framework to correct for the imperfect Se and Sp of the sampling scheme. Furthermore, a Se analysis was 
done to determine appropriate prior distributions for coefficients in the logistic regression model specifying region, housing type, and herd size. Distributions included in the Se analysis were centered on a herd-level prevalence of $50 \%$ (log odds of 0 , used as a mean for a normal distribution) based on an estimate for prevalence estimated in model 2 comparing $2 \mathrm{~S} 1 \mathrm{Y}$ and $3 \mathrm{~S} 1 \mathrm{Y}$. Values of precision varied between 0.0001 and 10,000 to represent very vague, weakly informative priors (low precision) and narrow, strongly informative priors (high precision), respectively. An intermediate level of precision was chosen that optimized the trade-off between high variability in the posterior distribution (results not interpretable or useful) and proximity of estimates to the prior (priors that are too strong may result in posterior estimates almost independent of observed data). Consequently, a weakly informative prior was chosen for all coefficients, centered on a prevalence of $50 \%$, with $95 \%$ probability of estimating a prevalence between 12.3 and $87.6 \%$ (Table 1). These parameters were estimated using a Gibbs sampler, with modes of resulting posterior distributions being used as the parameter estimates with their respective probability intervals. The model was run for the same number of iterations as the test accuracy model and convergence was monitored using the Gelman and Rubin R statistic (Gelman and Rubin, 1992).

Statistical significance of differences across characteristics (region, housing type, and herd size) was assessed using the apparent prevalence and maximum likelihood estimation in a logistic regression model using the glm function in base $\mathrm{R}$ ( $\mathrm{R}$ Core Team 2017). A P-value of $<0.05$ was considered significant. Models included either region, herd size, or housing type as predictors, with odds of testing MAP-positive as the outcome. Only farms categorized as freestall or tiestall were included in the housing-specific prevalence estimate models, as farms with bedded pack, other farms, or not specified were removed due to low sample sizes $(\mathrm{n}=9,1$, and 5 , respectively). Models with herd size and housing type as predictors were modeled using a mixed effects logistic regression with region as a random effect using the package lme4 (Bates et al., 2015). This mixed model adjusts estimates to account for unmeasured correlation between herds within a region.

\section{RESULTS}

\section{Herd Characteristics}

Of the 362 dairy farms across Canada participating in the NDS on-farm assessments, 55 (15\%) were in Western Canada (20 in British Columbia, 16 in Alberta, 9 in Manitoba, and 10 in Saskatchewan), 131
(36\%) in Ontario, 117 (32\%) in Québec, and 59 (16\%) in the Atlantic provinces (18 in Nova Scotia, 17 in New Brunswick, 20 in Prince Edward Island, and 4 in Newfoundland; Table 2).

In Western Canada, Ontario, and Atlantic Canada, the majority of participating farms housed their cows in freestalls (82, 54, and 59\%; respectively), whereas in Québec the majority of farms housed cows in tiestalls (73\%; Table 2). Across all 4 regions, freestall farms had a larger average herd size than tiestall farms $(P<$ 0.001; Table 2).

\section{Environmental Sample Results}

In all 4 regions across Canada, a higher number of MAP-positive environmental samples was collected from MSA than either LAC or BAH areas (Table 3). When BAH environmental samples for identification of MAP-positive farms were included, 4 additional farms from Ontario and Québec were MAP-positive. These farms included both tiestall and freestall farms and all herd size categories except for $>150$ cows (Table 3 ).

\section{Prior Test Characteristics}

Of the 62 farms (Wolf et al., 2014) with 6 samples collected in 3 consecutive years, $45(73 \%)$ had at least 1 positive environmental sample during at least 1 of the 3 sampling events (6S3Y). Estimated Se and Sp for the $6 \mathrm{~S} 3 \mathrm{Y}$ method for diagnosis of these herds were 93 and $98 \%$, respectively (Table 4 ). When collecting only 2 environmental samples from a herd at a single time point, Se of detecting MAP-infected herds was estimated at $40 \%$ and Sp to be $99 \%$ (2S1Y; Table 4).

\section{Estimating MAP Prevalence}

On the 362 dairy farms sampled for the NDS, Se and Sp were estimated at 38 and $100 \%$, respectively, when collecting 2 environmental samples (LAC and MSA; 2S1Y). These estimates did not change meaningfully when the third environmental sample from BAH was included (3S1Y; Table 5).

\section{Region, Housing Type, and Herd Size}

True herd-level MAP prevalence for all of Canada was $46 \%$, with true regional prevalence ranging from $24 \%$ in Québec to $66 \%$ in Western Canada (Table 6). The apparent prevalence varied for herd sizes, with herds having 101 to 150 and $>151$ cows having a higher apparent prevalence than herds with $\leq 50$ cows (Table 6 ). Additionally, across Canada, tiestalls had a lower apparent MAP prevalence than did freestalls (Table 
Table 2. Housing and herd size of the 362 farms sampled across Canada for the Canadian National Dairy Study

\begin{tabular}{|c|c|c|c|}
\hline Region $^{1}$ & Housing type & $\begin{array}{c}\text { No. of } \\
\text { herds (\%) }\end{array}$ & $\begin{array}{c}\text { Average adult } \\
\text { herd size (range) }\end{array}$ \\
\hline \multirow{5}{*}{ Western Canada } & & 55 & $166(40-802)$ \\
\hline & Freestall & $45(82)$ & $170(40-802)$ \\
\hline & Tiestall & $4(7)$ & $69(47-130)$ \\
\hline & Bedded pack & $1(2)$ & $234\left(\mathrm{NA}^{2}\right)$ \\
\hline & Not specified & $5(9)$ & $188(91-319)$ \\
\hline \multirow[t]{5}{*}{ Ontario } & & 131 & $90(16-600)$ \\
\hline & Freestall & $71(54)$ & $129(33-600)$ \\
\hline & Tiestall & $55(42)$ & $43(16-106)$ \\
\hline & Bedded pack & $4(3)$ & $56(35-72)$ \\
\hline & Not specified & $1(1)$ & 69 (NA) \\
\hline \multirow[t]{3}{*}{ Québec } & & 117 & $62(7-261)$ \\
\hline & Freestall & $32(27)$ & $96(37-261)$ \\
\hline & Tiestall & $85(73)$ & $49(7-130)$ \\
\hline \multirow{4}{*}{ Atlantic Canada } & & 59 & $84(13-429)$ \\
\hline & Freestall & $35(59)$ & $110(22-429)$ \\
\hline & Tiestall & $20(34)$ & $47(22-150)$ \\
\hline & Bedded pack & $4(7)$ & $42(13-70)$ \\
\hline
\end{tabular}

${ }^{1}$ Western Canada: British Columbia (20 herds), Alberta (16), Saskatchewan (10), and Manitoba (9); Atlantic Canada: New Brunswick (17 herds), Nova Scotia (18), Prince Edward Island (20), and Newfoundland (4).

${ }^{2} \mathrm{NA}=$ not applicable.

$6)$. Herd sizes in freestalls differed from tiestalls $(P<$ $0.001)$ and bedded packs $(P=0.03)$ when not adjusting for region. All other comparisons of herd size among housing types did not differ from each other.

\section{DISCUSSION}

In the current study, environmental sampling was used to determine herd-level prevalence of MAP infection in Canadian dairy herds. Sensitivity and Sp for culturing 2 environmental samples, followed by detec- tion of MAP based on F57-specific qPCR for detection of a positive farm, were estimated at 38 and $100 \%$, respectively. Based on these test characteristics, true prevalence across Canada was 46\%, with Québec having a lower prevalence than the other 3 regions. Although these estimations were not weighted by number of herds in each region, number of herds sampled in each region was proportional to the number in context of the entire Canadian dairy industry. This was the first time that the prevalence of MAP infection was determined using the same sampling technique across

Table 3. Herds with Mycobacterium avium ssp. paratuberculosis (MAP)-positive environmental samples collected from lactating cows (LAC), manure storage (MSA), or breeding-age heifers (BAH)

\begin{tabular}{|c|c|c|c|c|c|c|c|}
\hline Item & $\begin{array}{c}\text { No. } \\
\text { farms }\end{array}$ & $\begin{array}{l}\text { No. }(\%) \\
\text { LAC } \\
\text { positive }\end{array}$ & $\begin{array}{l}\text { No. }(\%) \\
\text { MSA } \\
\text { positive }\end{array}$ & $\begin{array}{l}\text { No. }(\%) \\
\text { BAH } \\
\text { positive }\end{array}$ & $\begin{array}{l}\text { No. }(\%) \text { farms } \\
\text { positive based on } \\
\text { LAC and MSA }\end{array}$ & $\begin{array}{l}\text { No. }(\%) \text { farms } \\
\text { positive based on LAC, } \\
\text { MSA, and BAH }\end{array}$ & $\begin{array}{c}\text { No. additional farms } \\
\text { due to MAP-positive } \\
\text { BAH sample }\end{array}$ \\
\hline \multicolumn{8}{|l|}{ Region $^{1}$} \\
\hline Ontario & 131 & $21(16)$ & $24(18)$ & $8(6)$ & $30(23)$ & $33(25)$ & 3 \\
\hline Québec & 117 & $4(3)$ & $7(6)$ & $2(2)$ & $11(9)$ & $12(10)$ & 1 \\
\hline Atlantic Canada & 59 & $7(12)$ & $8(13)$ & $1(1)$ & $11(19)$ & $11(19)$ & 0 \\
\hline Tiestall & 164 & $5(3)$ & $11(7)$ & $3(2)$ & $14(9)$ & $16(10)$ & 2 \\
\hline Bedded pack & 9 & $1(11)$ & $1(11)$ & 0 & $1(11)$ & $1(11)$ & 0 \\
\hline Other & 1 & 0 & 0 & 0 & 0 & 0 & 0 \\
\hline Not specified & 5 & $4(80)$ & $3(60)$ & 0 & $4(80)$ & $4(80)$ & 0 \\
\hline \multicolumn{8}{|l|}{ Number of cows } \\
\hline$<51$ & 141 & $7(5)$ & $11(8)$ & $4(3)$ & $15(9)$ & $17(12)$ & 2 \\
\hline $51-100$ & 125 & $10(8)$ & $9(7)$ & $2(1)$ & $13(10)$ & $14(11)$ & 1 \\
\hline
\end{tabular}

${ }^{1}$ Western Canada: British Columbia (20 herds), Alberta (16), Saskatchewan (10), and Manitoba (9); Atlantic Canada: New Brunswick (17 herds), Nova Scotia (18), Prince Edward Island (20), and Newfoundland (4). 
Table 4. Prior sensitivity and specificity estimates of herd-level Mycobacterium avium ssp. paratuberculosis (MAP) infection status based on at least 1 positive sample out of 6 collected every year for $3 \mathrm{yr}(6 \mathrm{~S} 3 \mathrm{Y})$ and MAP infection status based on minimally 1 positive sample of 2 collected in 1 yr $(2 \mathrm{~S} 1 \mathrm{Y})$

\begin{tabular}{lcc}
\hline Parameter & Estimate $^{1}$ & $95 \% \mathrm{CI}^{2}$ \\
\hline 6S3Y & 0.93 & $0.79-0.99$ \\
Sensitivity & 0.98 & $0.88-1.00$ \\
Specificity & 0.40 & $0.28-0.55$ \\
2 S1Y & & $0.81-1.00$ \\
Sensitivity & 0.99 & \\
Specificity &
\end{tabular}

${ }^{1}$ Estimate of the mode of the posterior distribution defined using a Bayesian model.

${ }^{2} 95 \%$ credibility interval from a posterior distribution using a Bayesian model. Represents the range of the central $95 \%$ probability of the distribution.

${ }^{3}$ Consisting of a manure storage and a lactating cow area sample.

all provinces of Canada, enabling accurate comparisons not only of regional effects, but also of housing and herd size effects across the country.

Herd-level prevalence estimates are made around the world using various tests; however, in Canada, the Netherlands, and the United States, prevalence has been estimated using the same diagnostic test. Based on the diagnostic outcome of serum ELISA testing, prevalence estimates ranged from $9.8 \%$ in Ontario to
Table 5. Sensitivity and specificity of herd-level Mycobacterium avium ssp. paratuberculosis (MAP) infection status for estimation of prevalence in the Canadian National Dairy Study, based on at least 1 MAP-positive environmental sample out of 2 collected in $1 \mathrm{yr}(2 \mathrm{~S} 1 \mathrm{Y})$, compared with 1 positive environmental sample out of 3 collected in $1 \mathrm{yr}(3 \mathrm{~S} 1 \mathrm{Y})$

\begin{tabular}{lcc}
\hline Parameter & Estimate $^{1}$ & $95 \% \mathrm{PI}^{2}$ \\
\hline 2S1Y & 0.38 & $0.26-0.49$ \\
Sensitivity & 1.00 & $0.92-1.00$ \\
Specificity & & \\
3S1Y & & \\
Sensitivity & 0.39 & $0.27-0.52$ \\
Specificity & 0.98 & $0.90-1.00$ \\
True Canada herd-level prevalence & 0.46 & $0.29-0.75$ \\
\hline
\end{tabular}

${ }^{1}$ Estimate of the mode of the posterior distribution defined using a Bayesian model.

${ }^{2}$ Refers to $95 \%$ probability interval from a posterior distribution using a Bayesian model. Represents range of the central $95 \%$ probability of the distribution.

${ }^{3}$ Consisting of a manure storage and a lactating cow area and breeding-age heifer area sample.

$40 \%$ in Alberta, 17 to $28 \%$ in the United States, and $54 \%$ in the Netherlands (Muskens et al., 2000; Tiwari et al., 2006). Additionally, herd-level prevalence estimates have been reported for individual Canadian provinces based on serum ELISA (Van Leeuwen et al., 2001, 2005, 2006; Scott et al., 2006) and are summarized in Tiwari et al. (2006). However, ability to compare

Table 6. Herd-level prevalence of Mycobacterium avium ssp. paratuberculosis (MAP) infection in Canadian dairy herds in 2015 for 3 separate models, stratified by region, herd size, or housing type

\begin{tabular}{|c|c|c|c|}
\hline Predictor level & $\begin{array}{c}\text { Apparent prevalence } \\
(\%)^{1}\end{array}$ & $\begin{array}{c}\text { True prevalence } \\
(\%)^{2}\end{array}$ & $95 \% \mathrm{PI}^{3}$ \\
\hline Canada & 18.8 & 46.4 & $28.5-74.6$ \\
\hline \multicolumn{4}{|l|}{ Region } \\
\hline Western Canada ${ }^{4}$ & 29.1 & 65.8 & $36.8-89.6$ \\
\hline Ontario & 22.9 & 54.1 & $0.3-83.7$ \\
\hline Québec & $9.4^{5}$ & 23.6 & $9.6-48.5$ \\
\hline Atlantic Canada & 18.6 & 47.3 & $21.6-79.2$ \\
\hline \multicolumn{4}{|c|}{ Herd size (no. of lactating cows) } \\
\hline$\leq 50^{*}$ & 10.5 & 21.1 & $8.8-36.4$ \\
\hline $51-100$ & 10.3 & 20.7 & $8.5-37.0$ \\
\hline 101-150 & $33.3^{5}$ & 61.2 & $35.9-86.7$ \\
\hline$>150$ & $48.4^{5}$ & 79.9 & $56.9-93.9$ \\
\hline \multicolumn{4}{|l|}{ Housing type } \\
\hline Freestall $^{4}$ & 26.5 & 63.7 & $40.2-88.9$ \\
\hline Tiestall & $8.5^{5}$ & 20.8 & $7.5-39.8$ \\
\hline Bedded pack & 10.9 & -6 & - \\
\hline Other & 0 & - & - \\
\hline
\end{tabular}

${ }^{1}$ Proportion of farms with at least 1 of 2 environmental samples (manure storage and lactating cow area) being MAP-culture positive.

${ }^{2}$ Calculated by taking the logit-transformation of the model coefficients.

${ }^{3} 95 \%$ probability interval from a posterior distribution using a Bayesian model. Represents the range of the central $95 \%$ probability of the distribution.

${ }^{4}$ Referent.

${ }^{5}$ Different $(P<0.05)$ from apparent prevalence in reference group within predictor.

${ }^{6}$ Number of farms was too small (9 and 1 for bedded pack and other, respectively) to reliably estimate true prevalence. 
regions is extremely difficult due to the variability introduced by types of kits used, cutoff points, laboratory environments, sampling technique and processing, and number of test-positive animals used to define a MAP-positive herd (McKenna et al., 2005; Tiwari et al., 2006). Additionally, these estimates appeared to differ when compared with prevalence estimates made using various diagnostic tests in the United States and regions of Canada (Lombard et al., 2006; Wolf et al., 2014). Although Se and Sp of various diagnostic tests have been investigated (McKenna et al., 2005; Lavers et al., 2013, 2014; Arango-Sabogal et al., 2016), these test characteristics are often not applied to prevalence studies and only apparent prevalence is reported. Additionally, the population used to obtain prior distributions is often not representative of the population under study (Barkema et al., 2010). True prevalence estimates in the current study using environmental samples were higher than previous estimates based on serum ELISA testing across Canada (Tiwari et al., 2006); however, these low estimates were likely due to an underestimation of prevalence by overestimating the Se of the ELISA as a result of misclassifying MAP-infected cattle as negative (Whitlock et al., 2000; Dargatz et al., 2001; Tiwari et al., 2006).

Distribution of freestall and tiestall farms in Canada collected in our study corresponded to the distribution of these herd types in the country [available through the Canadian Dairy Information Centre (CDIC); dairyinfo.gc.ca]. In Western and Atlantic Canada, 88 and 50\%, respectively, of DHI-participating farms house adult cows in a freestalls (CDIC), similar to distributions of housing types in the current study (82 and 59\%, respectively). In Québec, 93\% of farms use tiestalls (CDIC), a greater percentage than in the current study $(73 \%)$, which may result in overestimation of prevalence in that province. In Ontario, however, the proportion of freestall farms is higher according to the CDIC (69\%) than in the curren study (42\%). These differences between CDIC housing distributions and what we acquired were likely an artifact due to random sampling and a smaller sample size and should be considered for future investigations regarding housing and regional differences. Average herd sizes for tiestall and freestall farms sampled in our study were, in general, lower than the distribution reported by CDIC for all 4 regions. Average herd size for farms sampled in the current study had, on average, 35 fewer cows on freestall farms and 18 fewer cows on tiestall farms than CDIC. These differences were unlikely to have a large effect on comparisons among regions, as all farms sampled in all regions had on average smaller herds than reported by CDIC; however, smaller average herd size may lead to an underestimation of herd-level prevalence within each region and also for Canada as a whole.

Québec has, with respect to number of lactating cows and farms, the largest dairy industry in Canada, the lowest apparent prevalence and true prevalence (9.4 and $23.6 \%$, respectively), and the highest proportion of tiestall farms (69\%; Bauman et al., 2018). Herd size is highly associated with housing type, as tiestall farms usually have $<100$ cows (Wolf et al., 2014); the lower prevalence estimates in in Québec are, thus, potentially attributable to housing type or herd size rather than region. Farms with smaller herds are less likely to test MAP-positive; therefore, the lower prevalence in tiestall farms may be due to the lower herd sizes on these farms, and results should be interpreted with caution. Perhaps the contact structure of the herd, in addition to different management practices, contributed to a lower prevalence in tiestall farms. Additionally, information regarding access to pasture was not obtained and should be considered in future research when investigating management practices and contact structure of herds and housing types. It is also possible that the Se of prevalence estimates obtained from tiestall herds may be further encumbered, as environmental samples may not accurately represent a pooled sample from the herd. Most tiestall barns have gutter scrapers that move manure to form a pile with much less mixing of manure, which may lead to decreased detection among smaller herds and tiestall herds. In contrast, environmental samples collected from MSA of freestalls have high Se due to accumulation of fecal material and long-term survival of MAP in the environment (Whittington et al., 2004; Berghaus et al., 2006; Lombard et al., 2006). However, the lower prevalence of MAP-infected tiestall herds may not have been due to decreased detection of environmental samples from MSA, as these sites alone detected 11 of the 14 MAP-positive tiestall farms in Canada in the current study (Table 3). Although the majority of literature regarding Se and $\mathrm{Sp}$ of environmental sampling has been conducted in freestall herds (Raizman et al., 2004; Berghaus et al., 2006; Donat et al., 2015), evidence exists that environmental sampling can be just as effective in tiestall herds requiring inclusion of sampling a manure storage site (Arango-Sabogal et al., 2016).

Moisture and direct sunlight do not affect survival of MAP; however, temperature fluctuations potentially affect viability (Whittington et al., 2004). In the current study, environmental samples were collected from deep within manure storage sites, not in direct contact with sunlight and not exposed to temperature fluctuations on the surface of the pile. Additionally, DNA methods of detection from environmental samples were used fol- 
lowing culture; therefore, any MAP bacteria that may not have survived, but accumulated in high numbers in the culture bottles, would still be detected with qPCR.

The MAP prevalence was highest in larger herds. Of herds with 101 to 150 cows, 33\% tested positive and $49 \%$ were identified as MAP-positive in herds with $>150$ cows. This was consistent with reports that environmental samples collected from larger herds were more likely to be positive (Wolf et al., 2015b). The higher prevalence of MAP-infected farms among larger herds may be due to a higher within-herd prevalence (Pillars et al., 2009) or the higher likelihood that at least 1 cow was shedding MAP. However, the cause of the association between herd size and within-herd prevalence is not known, and higher prevalence in larger herds may be due to differences in management practices for young stock and increased purchase of replacement heifers, thereby increasing risk of transmission (Wolf et al., 2015a).

Adding BAH samples to the standard LAC and MSA samples identified 4 additional farms as being MAP-positive. These farms were only positive based on the BAH sample and therefore would have been incorrectly identified as negative had this sample not been included; however, MAP-positive BAH samples more often occurred when either LAC or storage site samples were positive. Additionally, BAH MAP-positive samples were not specific to regions, housing, or herd size categories. Identification of MAP-infected herds should not rely only on BAH environmental samples; however, positive BAH samples may be considered an important indicator of potential transmission to and within young stock, as positive environmental samples have been identified in all ages of calves (Raizman et al., 2004; Pillars et al., 2009; Wolf et al., 2015c).

Sensitivity estimates for 2 environmental samples were lower than previous estimates for 6 samples, which was expected, as fewer samples collected increases the odds that a truly infected farm is missed. However, the estimated prevalence in Alberta based on 2 environmental samples $(70 \%)$ in the subset of 62 herds was nearly the same as previous estimates (Wolf et al., 2014) based on 6 environmental samples (68\%) in all 360 participating Alberta dairy herds, indicating the accuracy of the test characteristic estimates. Therefore, collecting 2 environmental samples, LAC and MSA, enabled reliable estimation of true herd-level MAP prevalence in a region when accounting for adjusted test characteristics and should be considered to obtain region-specific herd-level estimates in future studies. The relatively large probability intervals surrounding the prevalence estimate were likely due to the high degree of regional variation, unmeasured differences in management practices, and, presumably, other undefined study characteristics. Due to the decreased Se when collecting only 2 environmental samples, this method would not be adequate for determining the infection status of a particular herd. The addition of the BAH samples did not meaningfully affect Se estimates, likely because the majority of farms only had MAP-positive BAH samples when also testing positive to either LAC or MSA samples, or both. Regardless, inclusion of environmental samples of young stock housing areas should be considered for future control programs and risk assessments, as it may be an indicator of transmission among young stock.

The number of farms recruited for on-farm sampling was based on ability to detect common mastitis pathogens in bulk milk tank samples (Bauman et al., 2018). Because prevalence of herd-level MAP infection is similar to the bulk tank prevalence of the most common udder pathogen, Staphylococcus aureus, in Canadian dairy herds (46\%; Bauman et al., 2018), sample size calculation used was also appropriate for evaluation of herd-level MAP infection. However, because detection of MAP in the environment is sensitive to within-herd prevalence, low-MAP prevalence herds may have remained undetected in the 6S3Y sampling of Wolf et al. (2014) as well as 2S1Y from NDS sampling, resulting in an overestimation of the Se and an underestimation of true herd prevalence (Raizman et al., 2004; Lavers et al., 2013). Additionally, due to the relatively small number of farms sampled from each region (for this kind of statistical analysis), stratification based on 2 variables resulted in extremely low power and therefore was not possible.

A further limitation of the study was an inability to account for ways that region, herd size, and housing were linked. The variables could not be stratified by $>1$ variable, as the number of herds that would be categorized in each stratum was too small to make a meaningful difference. For example, Western Canada only had $4(7 \%)$ tiestall farms that were sampled, and herd sizes among these farms were variable. In Quebec, $85(73 \%)$ farms were tiestalls, with herd size ranging from 7 to 130. Therefore, our interpretation of housing differences should be viewed with caution due to the large amount of collinearity with region.

\section{CONCLUSIONS}

Prevalence of MAP infection across Canada was estimated based on the culture of 2 environmental samples from a proportional number of farms in each of the 4 regions within the country. True herd-level prevalence of MAP infection was estimated in Western Canada as $65.8 \%(95 \%$ probability interval $=36.8-89.6)$, Ontario as $54.1 \%$ (95\% probability interval $=0.3-83.7$ ), Québec as $23.6 \%$ (95\% probability interval $=9.6-48.5)$, and 
Atlantic Canada as $47.3 \%$ (95\% probability interval $=21.6-79.2)$, adjusting apparent prevalence based on Se (0.38) and Sp (1.00) estimates for culturing 2 environmental samples for detection of a MAP-infected farm. Prevalence estimates were compared among regions, herd sizes, and housing types, indicating a lower prevalence among tiestall herds in smaller herds and in Québec. Inclusion of BAH samples did not significantly change Se of the test or prevalence estimates.

\section{ACKNOWLEDGMENTS}

This study was supported by the Dairy Cluster 2 Research program through the Canadian Agri-Science Clusters Initiative (Dairy Farmers of Canada, Agriculture and Agri-Food Canada, Canadian Dairy Commission, and Natural Sciences and Engineering Research Council). The authors thank John Kastelic for editing the manuscript and Uliana Kanevets and Aaron Lucko (University of Calgary) for technical assistance processing samples in the laboratory. This work was supported by the Natural Sciences and Engineering Research Council of Canada (NSERC) Industrial Research Chair in Infectious Diseases of Dairy Cattle (Alberta Milk, Edmonton, AB, Canada); Dairy Farmers of Canada (Ottawa, ON, Canada); Dairy Farmers of Manitoba (Winnipeg, MN, Canada); Westgen Endowment Fund (Abbotsford, BC, Canada); BC Dairy (Burnaby, BC, Canada); CanWest DHI (Guelph, ON, Canada); and Canadian Dairy Network (Guelph, ON, Canada).

\section{REFERENCES}

Arango-Sabogal, J. C., G. Cote, J. Pare, O. Labrecque, J. P. Roy, S. Buczinski, E. Dore, J. H. Fairbrother, N. Bissonnette, V. Wellemans, and G. Fecteau. 2016. Detection of Mycobacterium avium subspecies paratuberculosis in tie-stall dairy herds using a standardized environmental sampling technique and targeted pooled samples. Can. J. Vet. Res. 80:175-182.

Bakker, D. 2010. Paratuberculosis control measures in Europe. Pages 306-318 in Paratuberculosis: Organism, Disease and Control. M. A. Behr and D. M. Collins, ed. CABI, Wallingford, UK.

Barkema, H. W., J. W. Hesselink, S. L. McKenna, G. Benedictus, and H. Groenendaal. 2010. Global prevalence and economics of infection with Mycobacterium avium susbsp. paratuberculosis in ruminants. Pages 10-21 in Paratuberculosis: Organism, Disease, and Control. M. A. Behr and D. Collins, ed. CABI, Wallingford, UK.

Barkema, H. W., K. Orsel, S. S. Nielsen, A. P. Koets, V. Rutten, J. P. Bannantine, G. P. Keefe, D. F. Kelton, S. J. Wells, R. J. Whittington, C. G. Mackintosh, E. J. Manning, M. F. Weber, C. Heuer, T. L. Forde, C. Ritter, S. Roche, C. S. Corbett, R. Wolf, P. J. Griebel, J. P. Kastelic, and J. De Buck. 2018. Knowledge gaps that hamper prevention and control of Mycobacterium avium subspecies paratuberculosis infection. Transbound. Emerg. Dis. In press.

Bates, D., M. Machler, B. Bolker, and S. Walker. 2015. Fitting linear mixed-effects models using lme4. J. Stat. Softw. 67. https://doi .org/10.18637/jss.v067.i01

Bauman, C. A., H. W. Barkema, J. Dubuc, G. P. Keefe, and D. F. Kelton. 2018. Canadian National Dairy Study: Herd-level milk quality. J. Dairy Sci. 101:2679-2691.
Berghaus, R. D., T. B. Farver, R. J. Anderson, C. C. Jaravata, and I. A. Gardner. 2006. Environmental sampling for detection of Mycobacterium avium spp. paratuberculosis on large California dairies. J. Dairy Sci. 89:963-970.

Bolton, M. W., R. B. Pillars, J. B. Kaneene, W. A. Mauer, and D. L. Grooms. 2011. Detection of Mycobacterium avium subspecies paratuberculosis in naturally exposed dairy heifers and associated risk factors. J. Dairy Sci. 94:4669-4675.

Cocito, C., P. Gilot, M. Coene, M. de Kesel, P. Poupart, and P. Vannuffel. 1994. Paratuberculosis. Clin. Microbiol. Rev. 7:328-345.

Collins, M. T., V. Eggleston, and E. J. Manning. 2010. Successful control of Johne's disease in nine dairy herds: Results of a six-year field trial. J. Dairy Sci. 93:1638-1643.

Corbett, C. S., J. De Buck, K. Orsel, and H. W. Barkema. 2017. Fecal shedding and tissue infections demonstrate transmission of $M y$ cobacterium avium subsp. paratuberculosis in group-housed dairy calves. Vet. Res. (Faisalabad) 48:27.

Dargatz, D. A., A. B. Beverly, L. K. Barber, R. W. Sweeney, R. H Whitlock, W. P. Shulaw, R. H. Jacobson, and J. R. Stabel. 2001. Evaluation of a commercial ELISA for diagnosis of paratuberculosis in cattle. JAVMA 218:1163-1166.

Dendukuri, N., and L. Joseph. 2001. Bayesian approaches to modeling the conditional dependence between multiple diagnostic tests. Biometrics 57:158-167.

Denwood, M. J. 2016. runjags: An R package providing interface utilities, model templates, parallel computing methods and additional distributions for MCMC models inJAGS. J. Stat. Softw. 71:1-25.

Donat, K., J. Kube, J. Dressel, E. Einax, M. Pfeffer, and K. Failing. 2015. Detection of Mycobacterium avium subspecies paratuberculosis in environmental samples by faecal culture and real-time PCR in relation to apparent within-herd prevalence as determined by individual faecal culture. Epidemiol. Infect. 143:975-985.

Garcia, A. B., and L. Shalloo. 2015. Invited review: The economic impact and control of paratuberculosis in cattle. J. Dairy Sci. 98:5019-5039.

Gelman, A., and D. B. Rubin. 1992. Inference from iterative simulation using multiple sequences. Stat. Sci. 7:457-472.

Kalis, C. H., M. T. Collins, H. W. Barkema, and J. W. Hesselink. 2004. Certification of herds as free of Mycobacterium paratuberculosis infection: actual pooled faecal results versus certification model predictions. Prev. Vet. Med. 65:189-204.

Kruschke, J. K. 2015. Doing Bayesian Data Analysis, Second Edition: A Tutorial with R, JAGS, and Stan. Academic Press, San Diego, CA.

Lavers, C. J., H. W. Barkema, I. R. Dohoo, S. L. McKenna, and G. P. Keefe. 2014. Evaluation of milk ELISA for detection of Mycobacterium avium subspecies paratuberculosis in dairy herds and association with within-herd prevalence. J. Dairy Sci. 97:299-309.

Lavers, C. J., S. L. McKenna, I. R. Dohoo, H. W. Barkema, and G. P. Keefe. 2013. Evaluation of environmental fecal culture for $M y-$ cobacterium avium subspecies paratuberculosis detection in dairy herds and association with apparent within-herd prevalence. Can. Vet. J. 54:1053-1060.

Lombard, J. E. 2011. Epidemiology and economics of paratuberculosis. Vet. Clin. North Am. Food Anim. Pract. 27:525-535.

Lombard, J. E., B. A. Wagner, R. L. Smith, B. J. McCluskey, B. Harris, J. B. Payeur, F. Garry, and M. D. Salman. 2006. Evaluation of environmental sampling and culture to determine Mycobacterium avium subspecies paratuberculosis distribution and herd infection status on US dairy operations. J. Dairy Sci. 89:4163-4171.

McKenna, S. L., G. P. Keefe, H. W. Barkema, J. McClure, J. A. VanLeeuwen, P. Hanna, and D. C. Sockett. 2004. Cow-level prevalence of paratuberculosis in culled dairy cows in Atlantic Canada and Maine. J. Dairy Sci. 87:3770-3777.

McKenna, S. L., G. P. Keefe, H. W. Barkema, and D. C. Sockett. 2005. Evaluation of three ELISAs for Mycobacterium avium ssp. paratuberculosis using tissue and fecal culture as comparison standards. Vet. Microbiol. 110:105-111.

McKenna, S. L., G. P. Keefe, A. Tiwari, J. A. VanLeeuwen, and H. W. Barkema. 2006. Johne's disease in Canada Part II: Disease 
impacts, risk factors, and control programs for dairy producers. Can. Vet. J. 47:1089-1099.

Muskens, J., H. W. Barkema, E. Russchen, K. van Maanen, Y. Schukken, and D. Bakker. 2000. Prevalence and regional distribution of paratuberculosis in dairy herds in the Netherlands. Vet. Microbiol. $77: 253-261$.

Pieper, L., U. S. Sorge, T. DeVries, A. Godkin, K. Lissemore, and D. Kelton. 2015. Comparing ELISA test-positive prevalence, risk factors and management recommendations for Johne's disease prevention between organic and conventional dairy farms in Ontario, Canada. Prev. Vet. Med. 122:83-91.

Pillars, R. B., D. Grooms, and J. B. Kaneene. 2009. Longitudinal study of the distribution of Mycobacterium avium ssp. paratuberculosis in the environment of dairy herds in the Michigan Johne's disease control demonstration herd project. Can. Vet. J. 50:1039-1046.

Plummer, M. 2016. Bayesian graphical models using MCMC. R package version 4-6. Accessed March 2018. https://cran.r-project.org/ package $=$ rjags.

R Core Team. 2017. R: A language and environment for statistical computing. R Foundation for Statistical Computing, ed, Vienna, Austria.

Raizman, E. A., S. J. Wells, S. M. Godden, R. F. Bey, M. J. Oakes, D. C. Bentley, and K. E. Olsen. 2004. The distribution of Mycobacterium avium ssp. paratuberculosis in the environment surrounding Minnesota dairy farms. J. Dairy Sci. 87:2959-2966.

Scott, H., O. Sorensen, J. Wu, E. Chow, K. Manninen, and J. A VanLeeuwen. 2006. Seroprevalence of Mycobacterium avium subspecies paratuberculosis, Neospora caninum, bovine leukemia virus, and bovine viral diarrhea virus infection among dairy cattle and herds in Alberta and agroecological risk factors associated with seropositivity. Can. Vet. J. 47:981-991.

Stevenson, M., T. Nunes, C. Heuer, J. Marshall, J. Sanchez, R. Thornton, J. Reiczigel, J. Robinson-Cox, P. Sebastiani, P. Johann, and M. Jay. 2017. epiR: Tools for the analysis of epidemiological data. $\mathrm{R}$ package version 0.9-93. Accessed March 2018. https://cran.r -project.org/package=epiR.

Tiwari, A., J. A. VanLeeuwen, S. L. McKenna, G. P. Keefe, and H. W. Barkema. 2006. Johne's disease in Canada Part I: Clinical symptoms, pathophysiology, diagnosis, and prevalence in dairy herds. J. Vet. Diagn. Invest. 47:874-882.

VanLeeuwen, J. A., L. Forsythe, A. Tiwari, and R. Chartier. 2005. Seroprevalence of antibodies against bovine leukemia virus, bovine viral diarrhea virus, Mycobacterium avium subspecies paratuberculosis, and Neospora caninum in dairy cattle in Saskatchewan. Can. Vet. J. 42:56-58.

VanLeeuwen, J. A., G. P. Keefe, R. Tremblay, C. Power, and J. Wichtel. 2001. Seroprevalence of infection with Mycobacterium avium subspecies paratuberculosis, bovine leukemia virus, and bovine viral diarrhea virus in Maritime Canada dairy cattle. Can. Vet. J. 42:193-198.

VanLeeuwen, J. A., A. Tiwari, J. Plaizier, and T. Whiting. 2006. Seroprevalences of antibodies against bovine leukemia virus, bovine viral diarrhea virus, Mycobacterium avium subspecies paratuberculosis, and Neospora caninum in beef and dairy cattle in Manitoba. Can. Vet. J. 47:783-786.

van Schaik, G., S. M. Stehman, Y. H. Schukken, C. R. Rossiter, and S. J. Shin. 2003. Pooled fecal culture sampling for Mycobacterium avium ssp. paratuberculosis at different herd sizes and prevalence. J. Vet. Prev. Diag. 15:233-241.

Weber, M. F. 2006. Risk management of paratuberculosis in dairy herds. Ir. Vet. J. 59:555-561.

Whitlock, R. H., S. Wells, R. W. Sweeney, and J. Van Tiem. 2000 ELISA and fecal culture for paratuberculosis (Johne's disease): Sensitivity and specificity of each method. Vet. Microbiol. 77:387398.

Whittington, R. J., D. J. Marshall, P. J. Nicholls, I. B. Marsh, and L. A. Reddacliff. 2004. Survival and Dormancy of Mycobacterium avium ssp. paratuberculosis in the environment. Appl. Environ. Microbiol. 70:2989-3004.

Wolf, R., H. W. Barkema, J. De Buck, and K. Orsel. 2015a. Factors affecting management changes on farms participating in a Johne's disease control program. J. Dairy Sci. 98:7784-7796.

Wolf, R., H. W. Barkema, J. De Buck, and K. Orsel. 2015b. Sampling location, herd size, and season influence Mycobacterium avium ssp. paratuberculosis environmental culture results. J. Dairy Sci. 98:275-287.

Wolf, R., H. W. Barkema, J. De Buck, M. Slomp, J. Flaig, D. Haupstein, C. Pickel, and K. Orsel. 2014. High herd-level prevalence of Mycobacterium avium subspecies paratuberculosis in Western Canadian dairy farms, based on environmental sampling. J. Dairy Sci. 97:6250-6259

Wolf, R., K. Orsel, J. De Buck, and H. W. Barkema. 2015c. Calves shedding Mycobacterium avium subspecies paratuberculosis are common on infected dairy farms. Vet. Res. 46:71. 